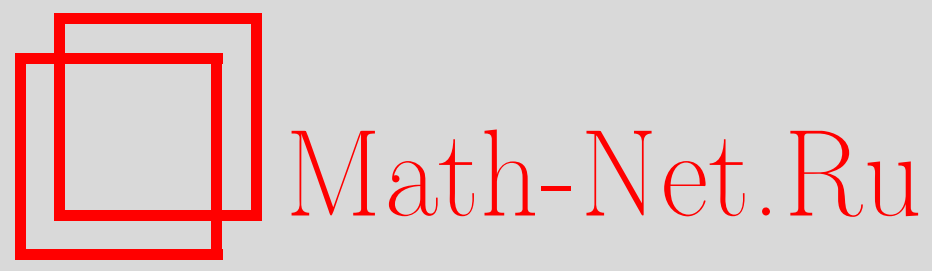

А. В. Бабаш, Внешне периодические автоматы, Дискрет. матем., 2005, том 17, выпуск 1, 68-72

DOI: https://doi.org/10.4213/dm88

Использование Общероссийского математического портала Math-Net.Ru подразумевает, что вы прочитали и согласны с пользовательским соглашением http://www . mathnet.ru/rus/agreement

Параметры загрузки:

IP : 54.198 .64 .247

26 апреля 2023 г., 15:25:59

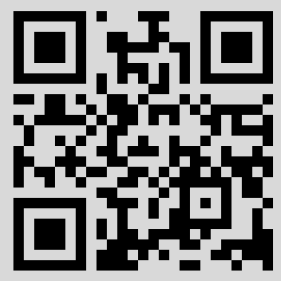


Удк 519.7

\title{
Внешне периодические автоматы
}

\author{
(c) 2005 г. $\quad$ A. В. Бабаш
}

Описываются автоматы, которые при любом начальном состоянии перерабатывают любую входную чисто периодическую последовательность в выходную чисто периодическую последовательность. Указан алгоритм, проверяющий, обладает ли данный автомат таким свойством. Введены и оценены сверху параметры его сложности.

Пусть $A=\left(X, S, Y,\left(h_{x}\right)_{x \in X},\left(f_{x}\right)_{x \in X}\right)$ - конечный автомат с входным алфавитом $X$, выходным алфавитом $Y$, множеством состояний $S$, частичными функциями перехода $\left(h_{x}\right)_{x \in X}$ и частичными функциями выхода $\left(f_{x}\right)_{x \in X}$. Введем следующие обозначения: пусть $|P|-$ длина входного слова $P$ автомата, $|S|$ - мощность множества состояний $S$ автомата $A$, $A(s, P)$ и $P(s)$ - выходное слово автомата $A$ длины $|A(s, P)|=|P|$ и его заключительное состояние, полученные в результате подачи входного слова $P$ на автомат $A$ с начальным состоянием $s \in S$, наконец, $\omega \mid L$ означает, что $\omega$ делит $L$. Под периодом периодической последовательности понимается ее минимальный период.

Определение 1. Автомат $A$ называется автоматом с $L$-потерей информации о выходе, если существуют входное слово $P$ длины $|P|=L, L \geqslant 1$, и состояния $s_{1}, s_{2} \in S$, для которых имеют место соотношения

$$
\begin{gathered}
A\left(s_{1}, P\right) \neq A\left(s_{2}, P\right), \\
P\left(s_{1}\right)=P\left(s_{2}\right),
\end{gathered}
$$

а при любом входном слове длины, меньшей $L$, не существует состояний с указанным свойством. Очевидно, граф переходов автомата $A$ с $L$-потерей информации о выходе содержит диаграмму вида

$$
\begin{aligned}
& s_{1} \stackrel{P, A\left(s_{1}, P\right)}{\longrightarrow} s^{\prime}, \\
& s_{2} \stackrel{P, A\left(s_{2}, P\right)}{\longrightarrow} s^{\prime},
\end{aligned}
$$

где

$$
s^{\prime}=P\left(s_{1}\right)=P\left(s_{2}\right), \quad A\left(s_{1}, P\right) \neq A\left(s_{2}, P\right), \quad|P|=L .
$$

Определение 2. Автомат $A$, не являющийся автоматом с $L$-потерей информации о выходе ни при каком $L$, называется автоматом без потери информации о выходе.

Лемма 1. Пусть $A$-автомат с $L_{A}$-потерей информачии о выходе. Тогда

$$
L_{A} \leqslant \frac{1}{2}|S|(|S|-1)+1 \text {. }
$$


Доказательство. Пусть выполнены условия леммы 1 и $P, s_{1}, s_{2}$ таковы, что

$$
P=x_{1} x_{2} \ldots x_{L}, \quad|P|=L=L_{A}, \quad P\left(s_{1}\right)=P\left(s_{2}\right)=s^{\prime}, \quad A\left(s_{1}, P\right) \neq A\left(s_{2}, P\right),
$$

при этом, очевидно, $f_{x_{1}} s_{1} \neq f_{x_{1}} s_{2}$ и

$$
f_{x_{j}} h_{x_{j-1}} \ldots h_{x_{1}} s_{1}=f_{x_{j}} h_{x_{j-1}} \ldots h_{x_{1}} s_{2}
$$

для $j \in\{2, \ldots, L\}$. Более того, легко показать, что при любом $j \in\{1, \ldots, L-1\}$ состояния $h_{x_{j}} \ldots h_{x_{1}} s_{1}$ и $h_{x_{j}} \ldots h_{x_{1}} s_{2}$ различны. Поэтому для доказательства леммы достаточно показать, что в множестве $M=\left\{h_{x_{j}} \ldots h_{x_{1}} s_{1}, h_{x_{j}} \ldots h_{x_{1}} s_{2}\right\}_{j \in[1, L-1]}$ неупорядоченных пар различных состояний нет одинаковых пар. Предположим, что такие пары имеются. Именно, пусть, например, для некоторых $k(1)<k(2), k(1), k(2) \in\{1, \ldots, L-1\}$, имеют место соотношения

$$
\begin{aligned}
& h_{x_{k(1)}} \ldots h_{x_{1}} s_{1}=h_{x_{k(2)}} \ldots h_{x_{1}} s_{2}, \\
& h_{x_{k(1)}} \ldots h_{x_{1}} s_{2}=h_{x_{k(2)}} \ldots h_{x_{1}} s_{1} .
\end{aligned}
$$

Заметим, что случай

$$
\begin{aligned}
& h_{x_{k(1)}} \ldots h_{x_{1}} s_{1}=h_{x_{k(2)}} \ldots h_{x_{1}} s_{1}, \\
& h_{x_{k(1)}} \ldots h_{x_{1}} s_{2}=h_{x_{k(2)}} \ldots h_{x_{1}} s_{2}
\end{aligned}
$$

рассматривается аналогично.

Тогда для входного слова

$$
\bar{P}=x_{1} x_{2} \ldots x_{k(1)} x_{k(2)+1} \ldots x_{L}
$$

длины $L-k(2)+k(1)$ и состояний $s_{1}, s_{2}$ справедливы соотношения

$$
\begin{gathered}
\bar{P}\left(s_{1}\right)=\bar{P}\left(s_{2}\right), \\
A\left(s_{1}, \bar{P}\right) \neq A\left(s_{2}, \bar{P}\right),
\end{gathered}
$$

что противоречит условию леммы 1. Таким образом, лемма 1 доказана.

Лемма 2. Пусть $A=\left(X, S, Y,\left(h_{x}\right)_{x \in X}, \lambda\right)$ - автомат Мура с $L_{A}$-потерей информачии о выходе и $\left\{N_{y}, y \in Y\right\}-$ мочности его классов 1-неотличимых состояний,

$$
\sum_{y \in Y} N_{y}=|S|
$$

Тогда

$$
L_{A} \leqslant 1+\sum_{y \in Y} \frac{1}{2} N_{y}\left(N_{y-1}\right) .
$$

Доказательство. Воспользовавшись обозначениями, введенными при доказательстве леммы 1 , и сформулированными там утверждениями, заключаем, что множество $M$ может быть разбито на классы $M_{y}, y \in Y$, где $M_{y}$ представляет собой множество всех пар $(a, b) \in M$, для которых $\lambda(a)=\lambda(b)=y$. Очевидно, что

$$
\bigcup_{y \in Y} M_{y}=M,
$$


причем для мощностей $\left|M_{y}\right|$ классов $M_{y}$ справедливы оценки

$$
\left|M_{y}\right| \leqslant \frac{1}{2} N_{y}\left(N_{y}-1\right), \quad y \in Y,
$$

из которых вытекает утверждение леммы 2.

Определение 3. Автомат $A$ называется внешне периодическим (внутренне периодическим), если для любой его входной чисто периодической последовательности $P$ и любого его начального состояния $s \in S$ его выходная последовательность $A(s, P)$ (его последовательность состояний) является чисто периодической последовательностью.

Теорема 1. Следующие два условия эквивалентны:

(1) автомат А является внешне периодическим автоматом,

(2) автомат А является автоматом без потери информачии о выходе.

Доказательство. Пусть $A-$ внешне периодический автомат с $L$-потерей информации о выходе и $s_{1}, s_{2}, P=x_{1}, x_{2}, \ldots, x_{L}$ таковы, что

$$
A\left(s_{1}, P\right) \neq A\left(s_{2}, P\right), \quad P\left(s_{1}\right)=P\left(s_{2}\right) .
$$

Тогда для входной периодической последовательности

$$
\bar{P}=\bar{x}_{1} \bar{x}_{2} \ldots \bar{x}_{j} \ldots,
$$

где $\bar{x}_{j}=\bar{x}_{j+L}$ для любого $j$ и $\left(\bar{x}_{1}, \bar{x}_{2}, \ldots, \bar{x}_{L}\right)=\left(x_{1}, x_{2}, \ldots, x_{L}\right)$ последовательности $A\left(s_{1}, P\right), A\left(s_{2}, P\right)$ чисто периодические. Кроме того, очевидно, что, начиная с номера $L+1$, элементы таких последовательностей совпадают. Поэтому должны совпадать и их начальные элементы, то есть $A\left(s_{1}, P\right)=A\left(s_{2}, P\right)$, что противоречит начальному допущению. Таким образом, показано, что условие 1) влечет условие 2).

Предположим теперь, что автомат $A$ не является внешне периодическим. Пусть $P=x_{1} x_{2} \ldots x_{L}$, а входная последовательность $A$ имеет вид $R=P P \ldots P \ldots$ (конкатенация слов $P$ ). Очевидно, что последовательность $R$ периодическая некоторого периода $\omega \mid L$. Пусть $s_{1}$ - состояние автомата, при котором последовательность $A\left(s_{1}, R\right)$ не является чисто периодической. Обозначим через $A_{M}\left(s_{1}, R\right)=s_{1} s_{2} \ldots$ последовательность состояний автомата $A$, отвечающую входной последовательности $R$ и начальному состоянию $s_{1} \in S$. В ее подпоследовательности $s_{L+1} s_{2 L+1} s_{3 L+1} \ldots$ имеются одинаковые состояния, так как мощность множества состояний $S$ автомата $A$ конечна. Пусть, напримep,

$$
s_{L r(1)+1}=s_{\operatorname{Lr}(2)+1}, \quad r(2)>r(1) .
$$

Не ограничивая общности доказательства, будем считать, что $r(2)-r(1)>r(1)$. Обозначим $(P)^{k}$ конкатенацию $P P \ldots P$, состоящую из $k$ слов $P$. Несложно проверить, что выходная последовательность $A\left(s_{1}, R\right)$ автомата $A$ имеет вид

$$
\begin{array}{r}
A\left(s_{1}, R\right)=A\left(s_{1},\left(P^{r(1)}\right)\right) A\left(s_{1+L r(1)},(P)^{r(2)-2 r(1)}\right) A\left(s_{1+L(r(2)-r(1))},(P)^{r(1)}\right) \\
A\left(s_{1+L r(1)},(P)^{r(2)-2 r(1)}\right) A\left(s_{1+L(r(2)-r(1))},(P)^{r(1)}\right) \ldots
\end{array}
$$

Ясно, что

$$
A\left(s_{1},(P)^{r(1)}\right) \neq A\left(s_{1+L(r(2)-r(1))},(P)^{r(1)}\right),
$$


так как в противном случае последовательность $A\left(s_{1}, R\right)$ была бы чисто периодической последовательностью с периодом $\omega \mid L(r(2)-r(1))$.

Таким образом, установлено, что граф переходов автомата $A$ содержит диаграмму вида

$$
\begin{gathered}
s_{1} \stackrel{P^{r(1)}, A\left(s_{1}, P^{r(1)}\right)}{\longrightarrow} s^{\prime}, \\
s_{1+L(r(2)-r(1))} \stackrel{P^{r(1)}, A\left(s_{1+L(r(2)-r(1))}, P^{r(1)}\right)}{\longrightarrow} s^{\prime},
\end{gathered}
$$

где

$$
s^{\prime}=s_{1+\operatorname{Lr}(1)}=s_{1+\operatorname{Lr}(2)},
$$

то есть автомат $A$ является автоматом с $L$-потерей информации о выходе, $L \leqslant L r(1)$.

Из теоремы 1 непосредственно вытекает следующее утверждение.

Следствие 1. Автомат А является внутренне периодическим тогда и только тогда, когда его частичные функции переходов осуществляют биекчии $S$ в $S$, то есть автомат А является перестановочным.

Теорема 2. Автомат А является внешне периодическим тогда и только тогда, когда для любой его чисто периодической входной последовательности $P$ периода $\omega \leqslant|S|(|S|-1) / 2+1$ и любого состояния $s \in S$ последовательность $A(s, P)$ является чисто периодической последовательностью.

Доказательство. Предположим, что автомат $A$ не является внешне периодическим автоматом. Тогда, согласно теореме 1 , он является автоматом с $L_{A}$-потерей информации о выходе. В первой части доказательства теоремы 1 показано, что в этом случае существуют чисто периодическая последовательность $P$ периода $\omega \leqslant L_{A}$ и состояние $s \in S$, для которых последовательность $A(s, P)$ не является чисто периодической последовательностью. В то же время, согласно лемме 1 ,

$$
L_{A} \leqslant|S|(|S|-1) / 2
$$

откуда и следует утверждение теоремы 2.

Использование леммы 2 для автомата Мура $A$ позволяет аналогично теореме 2 доказать следующее утверждение.

Теорема 3. Автомат Мура А является внешне периодическим автоматом тогда и только тогда, когда для любого $s \in S$ и любой его чисто периодической последовательности $P$ nерuода

$$
\omega \leqslant 1+\sum_{y \in Y} \frac{1}{2} N_{y}\left(N_{y}-1\right)
$$

где $N_{y}$ - мочность классов 1-неотличимых состояний, последовательность $A(s, P)$ является чисто периодической последовательностью.

Следствие 2. Существует алгоритм, проверяющий, является ли заданный автомат внешне периодическим автоматом. 
Доказательство. Действительно, для автомата Мура $A$, согласно теореме 2 , число последовательностей $A(s, P)$ при периодической входной последовательности $P$ периода

$$
\omega(P) \leqslant \frac{1}{2}|S|(|S|-1)+1
$$

и начальном состоянии $s$ конечно. Следовательно, достаточно доказать существование алгоритма, проверяющего, является ли фиксированная последовательность $A(s, P)$ при

$$
\omega(P) \leqslant|S|(|S|-1) / 2+1
$$

и $s \in S$ чисто периодической последовательностью. Существование же такого алгоритма следует из того факта (см. [1]), что приведенная длина исследуемой последовательности $A(s, P)$ ограничена сверху величиной $|S| \omega(P)$.

\section{Список литературы}

1. Кобринский Н. Е., Трахтенброт Б. А., Введение в теорию конечных автоматов. Наука, Москва, 1962.

Статья поступила 05.06.2003. 